\title{
Tsafon
}

Revue d'études juives du Nord

77 | 2019

Contribution à l'histoire des traductions juives de la Bible hébraïque

\section{Concepts et locutions talmudiques concernant la traduction biblique}

Une approche juive de la traduction dans l'Antiquité

\section{Francine Kaufmann}

\section{OpenEdition \\ Journals}

\section{Édition électronique}

URL : https://journals.openedition.org/tsafon/1593

DOI : $10.4000 /$ tsafon. 1593

ISSN : 2609-6420

\section{Éditeur}

Association Jean-Marie Delmaire

\section{Édition imprimée}

Date de publication : 1 septembre 2019

Pagination : 7-24

ISSN : $1149-6630$

Référence électronique

Francine Kaufmann, «Concepts et locutions talmudiques concernant la traduction biblique », Tsafon [En ligne], 77 | 2019, mis en ligne le 12 septembre 2019, consulté le 24 juin 2021. URL : http:// journals.openedition.org/tsafon/1593; DOI : https://doi.org/10.4000/tsafon.1593 


\title{
Concepts et locutions talmudiques concernant la traduction biblique
}

\author{
Une approche juive de la traduction dans l'Antiquité
}

Francine Kaufmann*

L'un des sous-domaines largement explorés en traductologie est l'étude des métaphores et concepts utilisés dans la littérature pour décrire ou évoquer la traduction et l'acte de traduire. Le traducteur peut être considéré comme un "passeur», un "gardien du seuil» (censeur qui veut empêcher un texte, une œuvre, d'entrer dans une autre civilisation ou d'en sortir), une « servante » dont le texte original serait le maître. Il peut être comparé à l'exécutant qui offre son interprétation d'une partition musicale, au « sculpteur » qui dans un nouveau matériau donne une forme neuve au modèle d'origine, à l'«adaptateur » qui destine l'œuvre à un nouveau public. Mais il est aussi vu comme un « traître » ou un « agent double », un « fabulateur» ou un « falsificateur ». Quant à la traduction elle-même, on la considère comme une « belle infidèle », un « baiser donné à travers un voile», un masque, un tuyau, une simple canalisation. Elle est copie, transfert, déplacement, transmutation, etc.

Il existe déjà bien des livres, des articles, et même des sites internet ${ }^{1}$ qui se penchent sur ce phénomène. Mon propos est d'explorer ici un autre corpus, qui appartient à une civilisation éloignée, dans le temps et dans l'espace, de la tradition occidentale : la civilisation juive de l'Antiquité.

\footnotetext{
*Université Bar-Ilan, Ramat-Gan, Israël.

${ }^{1}$ Voir la base de données sur le site de l'université Lyon II « Trésors des Métaphores de la traduction », conçue par François Géal, Professeur de Littérature comparée : $\mathrm{http}$ ://recherche.univ-lyon2.fr/tmt/index.php?controller=display\&page $=5$
} 


\section{Le contexte historique}

Le corpus auquel je me réfère, rarement étudié sous l'angle des métaphores de la traduction, est le Talmud, vaste recueil d'enseignements oraux des académies rabbiniques de Judée et de Babylonie. Rédigé entre le $\mathrm{II}^{\mathrm{e}}$ et le $\mathrm{V}^{\mathrm{e}}$ siècle de l'ère vulgaire, il s'appuie sur une tradition orale plusieurs fois séculaire, mise à contre cœur par écrit lorsque les guerres et les révoltes des Juifs contre Rome échouent, que les exils ou la dispersion puis la persécution religieuse menacent de couper matériellement et spirituellement le fil de la transmission orale de maître à élève. Après le désastre de la Première Guerre des Juifs qui aboutit à la destruction du Second Temple en 70 et à la mort des derniers combattants de Massada en 73, puis surtout après l'échec de la révolte de Bar Kokhba en 135, les rabbins décrètent l'état d'urgence qu'ils justifient en s'appuyant sur un verset du Psaume 119 (v.126), détourné de son sens premier : Ète la'assote la-Hachem, hèfèrou Toratekha : « Le moment est venu d'agir pour l'Éternel, on a renversé ta Torah ». En se fondant sur ce principe compris comme signifiant qu'il vaut mieux enfreindre un principe de la Torah plutôt que de risquer de la voir disparaitre tout entière, Rabbi Chimon ben Lakich et son beau-frère Rabbi Yo'hanan bar Nappaha expliquent :

Il vaut mieux enlever une lettre de la Torah plutôt que la Torah tout entière ne soit oubliée en Israël (TB Témourah, 14b).

Il existait pourtant un principe strict qui exigeait de conserver par écrit ce qui avait été transmis par écrit et oralement ce qui avait été transmis de bouche à oreille. Le metourguemane, l'interprète du même Chimon ben Lakich, Rabbi Yehouda bar Na'hmani l'expliquait ainsi :

Il est écrit : «Consigne PAR ÉCRIT ces paroles » (Exode XXXIV, 27). Il est aussi écrit [dans le même verset] : «Car PAR LA BOUCHE, ces paroles » [al pi hadevarim haélé = oralement; mais aussi : selon ces paroles]. Est-ce une contradiction? Les paroles écrites, tu n'as pas le droit de les énoncer de mémoire, et les paroles orales, tu n'as pas le droit de les énoncer à partir de l'écrit. ${ }^{2}$

\footnotetext{
${ }^{2} T B$ Guittin $60 \mathrm{~b}$, traduit et souligné par moi. Voir un texte quasi-similaire dans $T B$ Temourah 14b. Sur l'oral et l'écrit lire Alexis Nouss, «L'interdit et l'inter-dit: la traduisibilité et le sacré », Carrefours de la traduction, TTR 1989, vol. 2, n 1, p. 83-85. En ligne : https:/www.erudit.org/fr/revues/ttr/1989-v2-n1-ttr1470/037034ar.pdf
} 
En tout cas, décision est prise, dès le $\mathrm{II}^{\mathrm{e}}$ siècle, de transgresser l'interdiction de mettre par écrit la Torah orale (l'interprétation reçue de la Torah écrite) et de préserver dans l'écriture une collection d'enseignements des grandes écoles rabbiniques de l'époque, les batey midrach (maisons d'étude), qui risquaient autrement d'être perdus. Ces enseignements, recueillis et mémorisés oralement sont colligés puis mis en forme en hébreu par le Nassi ${ }^{3}$ de l'époque, Rabbi Yehouda : c'est la Michna. Or la traduction biblique est conçue comme partie intégrante de la Torah orale, puisqu'elle s'appuie sur l'interprétation de la Torah écrite reçue « au Mont Sinaï » et qu'elle en transmet oralement la tradition séculaire de lecture. On recueille donc parallèlement, sous l'autorité morale de deux rabbins illustres (Rabbi Éliézer et Rabbi Yehochoua), les traductions bibliques ayant cours dans les deux langues principales parlées dans le monde juif (araméen au nord et grec au sud), destinées essentiellement à la lecture synagogale. Il est vrai que la transmission de la traduction reçue risque aussi d'être perdue puisque les Romains persécutent également les Metourguemanim (les interprètes qui assurent, auprès du lecteur à la synagogue, la traduction-interprétation de la section sabbatique de la semaine ou qui assistent le Maître dans son enseignement) $)^{4}$. La première version du Pentateuque araméen, le Targoum Onkelos, (attribué à un prosélyte dont l'araméen était la langue maternelle), devient vite canonique dès le $\mathrm{III}^{\mathrm{e}}$ siècle et se retrouve aujourd'hui encore imprimée dans de nombreux volumes du Pentateuque aux côtés de l'original. Il s'agit de la tradition d'interprétation araméenne, largement diffusée au nord de la Palestine, particulièrement en Babylonie. Elle est si prisée qu'on affirme que même si la Torah semble intraduisible, le Targoum Onkelos, «notre traduction », réussit à être fidèle et qu'il permet de comprendre des passages qui, sans lui, seraient restés obscurs.

Celui qui traduit un verset littéralement est un menteur; Celui qui ajoute au texte est un sacrilège et un blasphémateur. Alors qu'est-ce que traduire ? C'est notre targoum [Onkelos] (TB Kiddouchine 49 a). ${ }^{5}$

\footnotetext{
${ }^{3}$ Le Nassi est le président du Sanhédrin. Il dirige en binôme le peuple d'Israël avec le président du tribunal, le Av beith Din.

${ }^{4}$ L'un des «dix martyrs » suppliciés et mis à mort par les Romains, lors des persécutions d'Hadrien, est Houtspit le Metourguemane, prestigieux interprète de Rabban Gamliel II. Houtspit traduisait ses enseignements dans sa maison d'étude. En assistant à son supplice, Élisha ben Abouya perdit la foi (TB Kiddouchin 39b).

5 En hébreu: Hametarguem ketsourato harey zé badä̈y: «Celui qui traduit littéralement [selon la forme], est un menteur [un affabulateur] ». Vehamossif 'alav,
} 
Le Targoum Onkelos sur le Pentateuque est complété par une traduction araméenne des Prophètes (dont certains passages sont sélectionnés pour accompagner la lecture synagogale - sidra hebdomadaire - et sont connus sous le nom de Haphtara). Attribué par le Talmud à Yonathan ben Ouziel, le Targoum Yonathan sur les Prophètes est lui aussi mis par écrit au $\mathrm{II}^{\mathrm{e}}$ siècle et canonisé au $\mathrm{III}^{\mathrm{e}}$. De son côté, converti au judaïsme Aquila, neveu de l'empereur Hadrien (est-ce une légende?), produit en même temps, pour l'aire hellénistique, une nouvelle traduction grecque du Pentateuque plus conforme à la tradition d'interprétation rabbinique de l'époque, placée elle aussi sous l'autorité morale de Rabbi Éliézer et Rabbi Yehochoua. Le Talmud de Jérusalem rapporte que ces rabbins l'encensent en se livrant à deux jeux de mots : kilssou oto (ils louèrent Aquilas dont le nom hébraïque évoque la racine : le-Kaless, louer) d'avoir si bien traduit la Bible en grec, dans la langue de Japhet, père de Yavan (ancêtre éponyme de la Grèce). Ce faisant ils louent la langue grecque, yevanit, synonyme de leshon Yafet, considérée comme langue de la beauté, yofi :

Rabbi Éliézer et Rabbi Yehochoua le glorifièrent (kilessou) en lui disant : « Tu l'as emporté en beauté [yafyafita] sur tous les hommes » (TJ Meguila I, 71, col. 3, hal. 9). ${ }^{6}$

\section{Japhet dans les tentes de Sem}

Déjà, justifiant le choix du seul grec pour traduire la Bible entière (TB Meguila 9a), le Nassi Rabban Chimon ben Gamliel (dont l'existence est attestée entre 140 et 165 après J.C.), avait loué les traducteurs en grec en recourant au même jeu de mots, tout en légitimant le grec par une interprétation de la bénédiction de Noé à ses deux fils, Sem et Japhet qui l'avaient couvert dans sa nudité :

"Que Dieu mette au large Japhet (Yaft Elohim le-Yéfète) ! Qu'il réside dans les tentes de Sem » (Genèse 9, 27). Les paroles de Japhet seront dans les tentes de Sem » (TB Meguila 9b). ${ }^{7}$

harey zé me'harèf oumegadèf: «Celui qui ajoute [au texte] est injurieux et insultant ». La seule façon de bien traduire est de suivre la traduction autorisée d'Onkelos: Targoum didane, notre targoum.

${ }^{6}$ Citation de Psaume 45, 3: «Tu es le plus beau (yafyafita mi-) des enfants des hommes ", choisie parce qu'elle fait jeu de mots avec Yéfète, l'ancêtre des Grecs.

${ }^{7}$ Voir aussi le Midrach Rabba sur Deutéronome, paracha 1, où le texte de Chimon ben Gamliel est un peu différent : «(Japhet) résidera dans les tentes de Sem de sorte que les 
Mais l'assonance entre Yaft (futur du verbe PTH, liftote, lehaftote) et yafyoute (la grâce, l'esthétique, le charme) induit l'interprétation donnée dans le même passage par rabbi Hiyya bar Abba qui comprend ainsi Yaft Elohim le-yéfète: «La beauté (yafyouto) de Japhet sera dans les tentes de Sem » (Ibid.).

On constate que l'élégance de la langue importe beaucoup aux maîtres du Talmud qui se rallient à l'opinion de Chimon ben Gamliel. Il est bénéfique de pouvoir embellir la Torah en la parant des beautés de la langue grecque, langue des belles lettres ${ }^{8}$. D'ailleurs le même Chimon ben Gamliel va plus loin. Il précise (dans le Talmud de Jérusalem qui traite des mêmes sujets) :

Car après examen, on a observé que le texte de la Torah peut être traduit le plus adéquatement en grec (TJ Meguila I, 9). ${ }^{9}$

Le grec permettrait donc une plus grande fidélité que d'autres langues à la transmission du message de la Torah. Il est vrai que la spécificité de chaque langue est évaluée par les rabbins. Ainsi Rabbi Yonathan de Beyt-Gouvrine, affirme :

Il y a quatre langues dont il sied que le monde fasse usage : le grec (laaz) pour le chant [ou la poésie], le latin (romi) pour la polémique [ou la guerre, la bataille], le syriaque (l'araméen, sursi) pour l'élégie [ou la lamentation], l'hébreu ('ivri) pour la parole ; certains ajoutent : et l'assyrien pour l'écriture (TJ Meguila I, 7 et Sotah 7, 2, 21c)

paroles de Sem seront dites dans les langues de Japhet. C'est pourquoi on a permis qu'elles soient écrites en langue grecque » (ma traduction). Voir enfin le Midrach Rabba sur Genèse, paracha 9: Bar Kappara disait clairement qu'il s'agissait d'une traduction biblique destinée au peuple d'Israël : «que les paroles de la Torah soient dites dans la langue de Japhet à l'intérieur des tentes de Sem (ma traduction) ».

${ }^{8}$ Rappelons qu'Ezra le scribe n'avait pas hésité lui aussi à emprunter un bien culturel à la puissante civilisation au sein de laquelle il résidait avant de retourner à Sion. Il avait remplacé l'alphabet paléo-hébraïque, proche du phénicien, par l'alphabet «syrien », dit « écriture carrée », en usage chez les Babyloniens. Mais Il faut remarquer que tous les rabbins n'étaient pas de l'avis de Rabban Chimon. Le débat était vif en ce qui concerne l'usage de langue grecque, surtout parce qu'elle était le vecteur de la philosophie grecque dont l'influence était considérée comme pernicieuse (cf. Mena'hot 99 b, Baba Kama 82 b - 83 a). Les modérés disaient : " Il faut distinguer entre la langue grecque et la sagesse grecque » (Baba Kama 83 a).

${ }^{9} \mathrm{Ou}$ : «Car après examen, on a constaté que la Torah ne peut être traduite de manière vraiment satisfaisante qu'en grec » (ma traduction). En hébreu : Badekou oumats'ou chéeyn haTorah yekhola lehitarguèm kol tsorka èla yevanite. 
La traduction est donc considérée comme un enrichissement et non comme un appauvrissement, surtout si, comme le grec, elle est susceptible de traduire la Torah « adéquatement» (kol tsorka: selon tous ses besoins). Naomi Seidman a raison de faire remarquer qu'en légitimant la résidence de Japhet chez Sem, les rabbins mettent l'accent sur l'importation des beautés inhérentes à une culture étrangère pour enrichir la culture de l'original, contrairement à la direction habituelle des transferts linguistiques (transport, déplacement du texte source exporté vers l'étranger). La traduction de la Bible en grec est considérée comme un « gain » pour le texte source, écrit-elle, non comme une perte. Sem profite des beautés de Japhet (et la culture juive s'enrichit des qualités de la langue et de la culture hellénistiques), mais en les « convertissant » à l'hébraïsme, de même qu'Aquila est devenu juif ${ }^{10}$.

Tous ne sont pas d'accord. Le passage du Talmud qui cite l'opinion de Rabban Chimon s'achève sur l'avis (rejeté il est vrai par les rabbins) de Rabbi Yehouda Bar Ilaï qui s'oppose à toute traduction sauf pour le Pentateuque qui peut être traduit mais seulement en grec, à cause du précédent miraculeux de la Septante où les traducteurs ont été inspirés par Dieu, ou comme le dit Rabbi Yehouda : «à cause de l'épisode du roi Ptolémée » (TB Meguila 9 a). En effet, bien qu'isolés dans 72 maisonnettes par celui qui leur avait commandé la traduction, Ptolémée d'Égypte, les traducteurs avaient modifié chacun de leur côté les passages ou les mots susceptibles d'aboutir à un malentendu, à un blasphème ou à un désagrément pour le peuple juif ${ }^{11}$. Les quinze exemples donnés par le Talmud sont édifiants: la traduction doit s'adapter à la civilisation du lecteur-cible en se mettant à sa place pour éviter qu'il ne se méprenne sur une formulation ambiguë ou inintelligible $^{12}$. Tenant compte des réflexes de lecture du public cible (Ptolémée et son peuple), les traducteurs ont fait usage d'un principe de précaution, évitant de reproduire certaines images ou formes syntaxiques que le lecteur de l'original comprend sans peine mais qui, une fois

\footnotetext{
${ }^{10}$ Lire Naomi Seidman, Faithful Renderings : Jewish-Christian Difference and the Politics of Translation, University of Chicago Press, 2006, p. 93. Elle consacre notamment son chapitre 2 à «La beauté de la Grèce dans les tentes de Sem. Aquila entre deux camps », p. 73-114.

${ }^{11}$ Le Talmud ne dit pas que tous les traducteurs ont abouti à une même traduction, comme le racontent Philon et Aristée, mais qu'ils ont tous évité les mêmes écueils.

12 J'ai longuement étudié ce passage du Talmud sur la Septante : Francine Kaufmann, « Un exemple d'approche théologique de la traduction : les jugements sur la Septante », dans La traduction des textes sacrés : le domaine biblique, TTR, 1990, 3 (2), 3-51. En ligne : https://www.erudit.org/fr/revues/ttr/1990-v3-n2-ttr1473/037067ar.pdf.
} 
traduites littéralement, risquent de suggérer une lecture polythéiste ou de laisser apparaître des incohérences. Dans le cas de Ptolémée, la traduction est conçue pour un public étranger (traduction centripète ou traduction exportée), tandis que pour un public juif à la synagogue, la traduction aurait dû suivre l'original verset par verset, tout en jouissant de la souplesse de l'oralité.

Mais on sait qu'avec le temps, l'hellénisme et la philosophie grecque ont été considérés comme menaçant la spiritualité juive. Avec la naissance du christianisme qui a adopté la Bible hébraïque non dans l'original mais dans la traduction grecque largement hellénisée des Septante, la lune de miel du judaïsme avec la Grèce s'achève. Non seulement les rabbins ne veulent plus que Japhet réside dans les tentes de Sem mais ils comparent au contraire les dégâts causés par la Septante à ceux causés par le culte idolâtre du veau d'or, renversant même le jugement des siècles précédents sur les vertus de la langue grecque :

Le jour où la Torah fut traduite en grec pour le roi Ptolémée fut aussi dur pour Israël que le jour où le veau d'or fut fabriqué PUISQUE LA TORAH NE POUVAIT PAS ÊTRE TRADUITE ADÉQUATEMENT. ${ }^{13}$ [Je souligne].

Un jeûne est même institué le 10 Tebeth pour commémorer ce malheur, inscrit dans un texte tardif de l'annexe hébraïque du « Rouleau des Jeûnes », Megillat Taanit ${ }^{14}$ : «Le 8 du mois de Tebeth, la Torah a été écrite en grec à l'époque du roi Ptolémée et les ténèbres sont descendus sur le monde durant trois jours » (ma traduction). Il me semble que la source de cette image se trouve dans le rapprochement fait entre les malheurs du peuple d'Israël dans l'Égypte du Pharaon et ceux engendrés par l'Égypte hellénisée. C'est sur l'île de Pharos qui fait face à Alexandrie que la traduction fut effectuée et qu'une fête était célébrée durant des siècles. Les trois jours de ténèbres évoquent la neuvième plaie

\footnotetext{
${ }^{13}$ Sefer Torah I, 8 - III ${ }^{\mathrm{e}}$ siècle; Massekhet soferim I, 7, milieu du VIII ${ }^{\mathrm{e}}$ siècle. En hébreu : Eyn haTorah yekhola lehitarguèm kol tsorka : «La Torah ne peut pas être traduite de manière satisfaisante »; voir plus haut l'affirmation inverse.

${ }^{14}$ Ce rouleau ne contient pas la liste des jours de jeûne mais au contraire celle des jours commémorant d'heureux événements, où il est interdit de jeûner. Rédigé en araméen, sans doute au $\mathrm{I}^{\mathrm{e}}$ siècle, il est clos par cette formule: "Ainsi s'achève la Meguillath Taanith» (à ne pas confondre avec le traité talmudique Taanith). Dans sa forme actuelle, il contient un addendum en hébreu : Maamar a 'harone (Postface) qui, de l'avis des savants, daterait du VIII ${ }^{\mathrm{e}}$ siècle. C'est là que figure cette phrase citée ici (Meguillath Taanith 13). Entre temps l'islam était né lui aussi au $\mathrm{VII}^{\mathrm{e}}$ siècle, sur le terreau de la Bible traduite en grec par les Septante.
} 
d'Égypte qui plongea le pays dans trois jours de ténèbres : «Moïse dirigea sa main vers le ciel et d'épaisses ténèbres couvrirent tout le pays d'Égypte, durant trois jours » (Exode 10, 22).

L'image d'une traduction qui produit des ténèbres s'oppose à l'image habituelle de la traduction qui clarifie et explique (bèour), comme nous allons le voir.

\section{Dire le traduire}

On aura déjà constaté que la traduction se confond dans l'Antiquité juive avec l'explication ou la reformulation intralinguale, l'interprétation orale : c'est le sens premier du verbe letarguèm dans le Talmud, et des significations attribuées par lui à certains vocables bibliques que nous exposerons ci-dessous comme ba'èr haytèv: bien expliqué, clarifié, meforach: expliqué, explicite. Mieux, l'acte de traduire est souvent rendu par les termes habituels qui désignent l'énonciation ou l'écriture. À propos d'Onkelos et de Yonathan ben Ouziel, le Talmud emploie pour chacun d'eux le verbe amar (a dit) pour leur traduction en araméen ${ }^{15}$. Les Septante «ont écrit» (katevou) la Torah en grec pour Ptolémée (TB Meguila 9 a et B). Les livres de la Bible peuvent "être écrits» (ketouvim) en toutes langues (Michna Meguila VIII, 9) ${ }^{16}$. Notons que dans TB Chabbat 115 a, il est question des Écrits saints «écrits »en copte, mède, élamite, grec, et en caractères hébraïques anciens qu'on doit sauver d'un incendie le jour du Chabbat, tout comme ceux « écrits » en targoum (au sens restreint en araméen) et en toute autre langue. Mais le concept targoum qui exprime d'abord l'idée d'interpréter un texte (c'està-dire de l'expliquer dans la même langue ${ }^{17}$ ou dans une langue étrangère) a fini par se spécialiser pour désigner, au sens large, toute

\footnotetext{
${ }^{15}$ Les maîtres du Talmud sont également appelés : Amoraïm (plur. de Amora, de la racine $A M R$ : dire, exposer, énoncer, qui a aussi le sens d'interpréter. Amora est tout d'abord un synonyme de Metourguemane et désigne celui qui interprète, celui qui explicite le sens des enseignements au Beyt Hamidrach (la maison d'études) ou à la synagogue.

16 Mais cette michna concerne peut-être uniquement la «transcription» ou «translittération » du texte biblique dans un autre alphabet, ce qui semble avoir été le cas à Alexandrie où la Torah hébraïque était lue dans un texte transcrit en caractères grecs, cf. Lévinas, «La traduction de l'écriture. Traité Meguila folio $8 \mathrm{~b}$ et $9 \mathrm{a}-9 \mathrm{~b}$ » dans Israël, le judaïsme et l'Europe, Actes du XXIII Colloque des intellectuels juifs de langue française, Paris, Gallimard, coll. Idées, 1984, p. 331-369. Voir aussi Genèse Rabba XXXVI et Deutéronome Rabba, 1.

${ }^{17}$ Dans le Talmud, « [Rabbi untel] tirguèm » signifie : [Rabbi untel] expliquait ainsi...
} 
traduction. Nous l'avons vu, l'amora (en Palestine), comme en Babylonie le metourguemane, ou tourguemane ${ }^{18}$ (d'après l'araméen), désigne l'interprète qui explicite l'enseignement du maître ou du prédicateur, mais si celui-ci vient d'une autre aire linguistique (maître babylonien venu enseigner en Judée ou vice-versa), sa tâche consiste aussi à effectuer un transfert linguistique en «traduisant » de la langue étrangère en langue locale.

Le premier metourguemane évoqué dans la Bible est Aaron. Son frère Moïse refuse la mission que Dieu lui confie parce qu'il est bègue, qu'il a la «langue embarrassée » (Exode IV, 10). Dieu lui adjoint alors un « interprète » :

Eh bien! ton frère Aaron, le Lévite, je sais que lui il parlera ! [...] Tu lui parleras, tu mettras les paroles dans sa bouche, et moi je serai avec ta bouche et avec sa bouche $[\ldots]$ C'est lui qui parlera pour toi au peuple; ainsi il te tiendra lieu de bouche, et toi tu lui tiendras lieu de elohim [Seigneur, inspirateur] (Exode $4,14-16)$.

Rachi explique le sens ad hoc de elohim par: mèlits, mot qui désigne l'interprète égyptien qui se tenait entre Joseph et ses frères (Genèse 42, 23). La racine signifie « bien parler, être doté d'éloquence ». L'interprète est un homme qui maîtrise la parole, qui sert de bouche, de porte-parole à son mandant. Le traducteur-interprète est une " bouche » qui dit, qui énonce. C'est sa fonction première.

Alors l'Éternel dit à Moïse : Regarde ! je fais de toi un dieu à l'égard de Pharaon et Aaron ton frère sera ton prophète (Exode 7, 1).

Onkelos traduit ici «prophète » par metourguemane. L'image est frappante. Il est probable qu'Aaron s'adressait en hébreu au peuple d'Israël et en langue égyptienne au Pharaon (cf. Exode 7, 2). Mais sa fonction essentielle consistait moins à traduire qu'à réexprimer de manière éloquente ce que son frère Moïse, handicapé du langage, ne pouvait formuler de manière convaincante.

À la fin de sa vie, Moïse expose (bèèr) la Torah devant le peuple entier (Deutéronome 1, 5). Puis il ordonne au peuple, quand il aura

\footnotetext{
${ }^{18}$ On constate le rapprochement avec les emprunts en français : «drogman », interprète des pays du Levant (du grec byzantin dragoumanos, par le biais de l'italien dragommanos), qui par rapprochement avec l'arabe tourdjouman a donné en français « truchement », interprète, intermédiaire.
} 
franchi le Jourdain, d'écrire sur de grandes pierres enduites de chaux toutes les paroles de la Torah très distinctement, bien clairement : baèr heytev, (Deutéronome 27, 8). Le verbe baer signifie : clarifier, expliquer. Le livre de Josué affirme que ceci fut fait, que Josué recopia la Torah de Moïse (Jos 8, 32) et qu'il en lut le texte en présence de tout Israël « et des étrangers qui vivaient parmi eux » (Jos 8, 33.35).

Pour le Talmud et les commentateurs, l'un des sens du baèr heytèv est que le texte doit être accompagné de son corollaire implicite, le bèour : une «clarification » pour ceux qui ne comprennent pas le sens du texte, une " traduction » pour les étrangers présents qui ne comprennent pas la langue du texte : "Vous écrirez sur les pierres toutes les paroles de cette Torah : écrit, gravé et bien expliqué, se lisant dans une langue et se traduisant dans 70 langues ${ }^{19}$ (Targoum Yonatan et TB Sota 36a, ma traduction). Le grand exégète champenois Rachi (1040-1105) choisit également cette interprétation du baèr heytèv de Dt 27, 8: «Bien clairement - en 70 langues ». Ceci correspond à l'interprétation qu'il avait donnée de la même expression figurant au premier chapitre du Deutéronome, lorsque Moïse avait commencé au pays de Moab d'expliquer la Torah devant tout Israël : «Bèèr : C'est en 70 langues qu'il la leur expliqua » (Rachi sur Dt 1, 5, ma traduction).

Avec le temps, un nouveau mot est introduit, avec un sens parallèle : meforach, (littéralement : expliqué ; du verbe lefarèch, mais aussi explicite); avec le corollaire de la traduction pour ceux qui ne comprennent pas la langue employée. On trouve ce nouveau vocable dans le récit de la première lecture publique de la Torah convoquée à Jérusalem par Ezra au retour de Babylone, autour de 400-380 av. J.-C. Beaucoup de Juifs ne savaient plus l'hébreu après 70 ans d'exil, certains avaient épousé des femmes étrangères des femmes d'Achdod, de Moab ou d'Amon et parlaient leur langue (Néhémie 13, 23-24). Ezra eut donc recours à l'interprétation en mêlant à la foule des mévinim (du verbe lehavine, comprendre, faire comprendre), des érudits qui aidaient la foule à suivre la lecture. « Ils lisaient dans le livre, dans la Torah de l'Éternel, meforach, de telle sorte que l'on puisse comprendre la lecture » (Néhémie 8,8 ). Selon Rab, un amora babylonien du III ${ }^{\mathrm{e}}$ siècle, le verset suggère que le peuple comprenait la lecture en hébreu parce qu'elle était

\footnotetext{
${ }^{19}$ La tradition illustre la multiplicité des peuples et des langues par le nombre 70 : les 70 langues renvoient aux 70 nations du monde qui représentent symboliquement toute l'humanité.
} 
rendue explicite par sa traduction. Ainsi, peut-on lire dans le traité Nedarim :

Rab a dit : Que signifie: «Ils lisaient dans le livre de la loi de Dieu, d'une manière distincte, et ils en indiquaient le sens, pour faire comprendre ce qu'ils avaient lu »? Le livre de la loi de Dieu, c'est le Texte lui-même ; d'une manière distincte [meforach] : c'est à dire en le traduisant; et ils en indiquaient le sens : allusion à la division en versets; pour faire comprendre ce qu'ils avaient $l u$ : allusion à la ponctuation; certains disent qu'il s'agit du texte traditionnel (TB Nedarim $37 \mathrm{~b}){ }^{20}$

Les mevinim transmettaient, avec la tradition de lecture et d'interprétation, à la fois le sens et le rythme du texte biblique. On apprend de là que le rôle primordial de la traduction est de clarifier le sens, de l'expliquer, car il ne suffit pas que l'auditeur entende la lecture rituelle de la Torah, il est nécessaire qu'il la comprenne.

\section{La traduction, un texte éclaté}

On pourrait poursuivre la description des métaphores et des images qui rendent compte de l'approche juive de la traduction dans l'Antiquité. Mais le cadre de cet article n'y suffit pas. Nous terminerons donc par l'une des plus belles, celle d'une parole divine éclatée aux quatre coins du monde, qui semble indiquer que l'entreprise de traduction des textes sacrés est inscrite, dès l'origine, dans le projet divin. La tradition juive enseigne en effet que lorsque la parole de Dieu s'adressa à Moïse et au peuple d'Israël au Mont Sinaï, la révélation fut polyglotte et polysémique :

Il est écrit : «Le peuple entier voyait les voix » (Exode 20,15); le mot « voix » est au pluriel. Rabbi Yo'hanan disait que toute voix sortait et se fractionnait (nè'hèlak) en sept voix et ces sept voix en soixante-dix langages (lachone) pour que toutes les nations entendent et que chaque nation entende Sa voix dans sa propre langue. ${ }^{21}$

\footnotetext{
${ }^{20}$ Aggadoth du Talmud de Babylone (la Source de Jacob - 'Ein Yaakov), traduit et annoté par Arlette Elkä̈m-Sartre, collection «Les Dix Paroles », Paris, Verdier. 1982, p. 680. La traductrice traduit le pluriel massoroth/les traditions, par «texte traditionnel ", induisant qu'il s'agissait de la version qu'on appellera plus tard "massorétique », texte découpé, ponctué et vocalisé conformément aux traditions orales de lecture par les massorètes, entre les $\mathrm{VI}^{\mathrm{e}}$ et $\mathrm{VII}^{\mathrm{e}}$ siècles. Voir aussi TB Meguilla $3 \mathrm{~b}$ et TJ Meguilla $1,2$.

${ }^{21}$ Tan'houma sur Exode, simane 25, parag. 5. Ce midrach s'appuie sur TB Chabbat 88

b. Cf. aussi Sifré sur Dt Vezoth Haberakha : «Lorsque Le Saint Béni-soit-Il se révéla
} 
À l'école de Rabbi Ismaël, l'un des maîtres de l'herméneutique juive ( $\mathrm{II}^{\mathrm{e}}$ siècle), on commentait ainsi ce verset du prophète Jérémie : « Ma parole n'est-elle pas comme un marteau qui fait voler en éclats le rocher? » (Jérémie 23, 29) :

De même que le roc vole en éclats sous les coups du marteau, de même chaque parole que Dieu a prononcée s'est fragmentée en soixante-dix langages (TB Chabbat 88 b).

Le même texte est repris dans un midrach, le Yalkout Chimoni (XIII ${ }^{\mathrm{e}}$ siècle) qui ajoute une seconde dimension à cette parabole de la transmission d'un message linguistiquement éclaté :

Rabbi Yehochoua ben Lévy dit: «C'est comme l'homme qui frappe sur l'enclume et les étincelles fusent de toutes parts »; Rabbi Yossey bar Hanina dit : «C'est comme l'homme qui frappe la pierre avec un marteau, et les éclats volent de toutes parts ».

Les traductions de la Torah (de la Bible hébraïque) dans les 70 langues du monde représentent donc des «projections » (des étincelles selon Rabbi Yehochoua) ou des «fragments» (des éclats selon Rabbi Yossey) de la Torah originale (parole divine révélée au Sinaï). Elles sont consubstantielles de l'original puisque la parole divine s'adresse à l'ensemble de l'humanité (les 70 nations), ou en tout cas à l'humanité connue dans l'Antiquité. On n'est pas loin des préoccupations de Walter Benjamin dans « La tâche du traducteur » sur la survie de l'original grâce à la traduction.

pour donner la Torah à Israël, il ne se révéla pas dans une seule langue, mais dans quatre : l'hébreu (Sinaï), le latin (Séïr), l'arabe (Paran) et l'araméen (rivevoth kodèch) ». 